\title{
The Bizarre Central Star of SuWt2
}

\author{
Katrina Exter ${ }^{1}$, Howard Bond ${ }^{2}$, Don Pollacco ${ }^{1}$, Philip Dufton ${ }^{1}$ \\ ${ }^{1}$ APS Division, Dept of Pure and Applied Physics, Queens University \\ Belfast, Belfast, BT7 1NN, UK \\ ${ }^{2}$ STScI, 3700 San Martin Drive, Johns Hopkins University Homewood \\ Campus, Baltimore, MD 21218 USA
}

\begin{abstract}
SuWt2 has been found to contain a double lined and eclipsing binary system. Surprisingly, both components appear to be A-type stars with masses of about $3 \mathrm{M}_{\odot}$ moving in essentially circular orbits with a period of 4.9 days. We see no indications of a hotter component in the optical or IUE spectra. We discuss the possibility that this is a triple system.

Deep $\mathrm{H} \alpha+[\mathrm{N}$ II] images show the nebula to be an inclined ring $\left(\sim 60^{\circ}\right.$ to the line of sight) while spectra show anomalous line ratios (eg $\mathrm{I}([\mathrm{N} \mathrm{II}] 6584)>>\mathrm{I}(\mathrm{H} \alpha))$ which maybe indicative of recombination in a changing radiation field. Further modeling is ongoing.
\end{abstract}

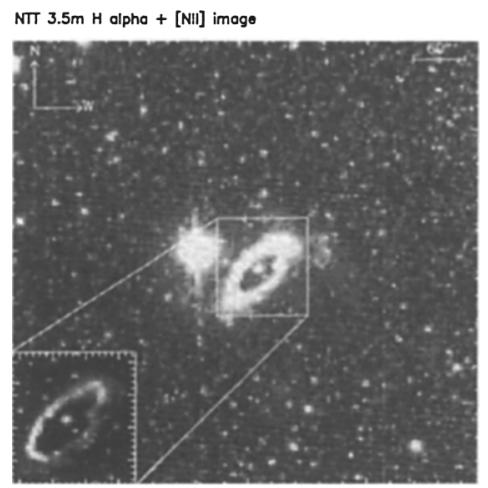

Figure 1. Narrow-band image of SuWt2. The faint bipolar lobes are only faintly visible on the original CCD image.



Figure 2. SuWt2 double lined radial velocity curve 